\title{
A Spectral Efficient NOMA-based Two-Way Relaying Scheme for Wireless Networks with Two Relays
}

\author{
Guosheng Li \\ School of Intelligent Information Engineering \\ Zhejiang Wanli University \\ Ningbo, 315100, China \\ [e-mail: nblgs@foxmail.com] \\ *Corresponding author: Guosheng Li \\ Received October 6, 2020; revised November 23, 2020; accepted December 19, 2020; \\ published January 31, 2021
}

\begin{abstract}
This paper proposes a novel two-way relaying (TWR) approach for a two-relay wireless network based on non-orthogonal multiple access (NOMA), where two terminals exchange messages with a cellular base station (BS) via two intermediate relay stations (RSs). We propose a NOMA-based TWR approach with two relaying schemes, i.e., amplify-and-forward (AF) and decode-and-forward (DF), referred to as NOMA-AF and NOMA-DF. The sum-rate performance of our proposed NOMA-AF and NOMA-DF is analyzed. A closed-form sumrate upper bound for the NOMA-AF is obtained, and the exact ergodic sum-rate of NOMADF is also derived. The asymptotic sum-rate of NOMA-AF and NOMA-DF is also analyzed. Simulation results show that the proposed scheme outperforms conventional orthogonal multiple access based transmission schemes. It is also shown that increasing the transmit power budget of the relays only cannot always improve the sum-rates.
\end{abstract}

Keywords: NOMA, Successive Interference Cancellation, Sum-Rate Performance, TwoWay Relay 


\section{Introduction}

The non-orthogonal multiple access (NOMA) technique has attracted a lot of research interests recently as a promising solution to meet the increasing demand for low latency and massive connectivity [1-3]. Compared with orthogonal multiple access (OMA), NOMA improves transmission efficiency by allowing multiple wireless users to share the same radio resources via superposition signaling.

Recently, the NOMA technique has been successfully applied in relay-assisted wireless communication systems [4-7]. The authors in [4] proposed a cooperative NOMA approach, where a user with better link quality was selected as a relay to forward the signals. In [5], the authors considered a two-user system that the full-duplex (FD) near user helps the far user, and the outage performance for two NOMA users was analyzed. For networks with dedicated relays, the authors in $[6,7]$ proposed relaying schemes that the relay station and the direct link user forms a NOMA pair, and the relay forwards signal to the indirect link user with decodeand-forward (DF) [6], or amplify-and-forward (AF) [7] relaying. For NOMA-based transmission with multiple relays, a relay selection approach can be employed to obtain spatial diversity gain [8-10]. For instance, based on the global channel information, a two-stage relay selection scheme was proposed in [10], and the outage probability as well as the diversity gain was analyzed.

All these aforementioned NOMA-based relaying schemes considered one-way relaying only. It is well known that two-way relaying (TWR) [11-13] is more efficient than one-way relaying. Hence, it is natural to combine NOMA with TWR technique [14-17]. In [16], the authors considered a NOMA two-way relay network (TWRN) and analyzed the outage performance. In [17], a hybrid two-way relaying scheme was proposed, which combined the NOMA and network coding techniques. The authors in [18] studied the spectral efficiency of NOMA-based TWRN with opportunistic relay selection. It was shown in [18] that the energy efficiency of NOMA-based TWR transmission can be greatly improved. In [19], a full-duplex DF relaying based cooperative NOMA TWR transmission was proposed to improve the system throughput.

For more sophisticated two-way relaying systems, such as multi pair TWRNs [20-22] and multi-way relay networks (MWRNs) [23, 24], the system throughput can also be improved by applying NOMA. In [20], the authors considered a NOMA multipair TWRN, in which multiple pairs of users communicate with each other with the help of several relay nodes. A rate splitting scheme with successive decoding was proposed to combat the interference. A cognitive radio inspired NOMA-based network coding scheme was proposed in [21] to improve the spectral efficiency of multipair TWRNs. In [22], the authors designed beamforming matrices for performance optimization in a NOMA-based multi-pair TWRN. In [24], the authors investigated an unmanned aerial vehicle (UAV)-aided NOMA MWRN. Numerical results showed that these proposed approaches [22-24] can significantly improve the sum-rates by reducing the required time slots for information exchange.

Most of these existing works [20-25] considered NOMA transmission with a single relay node only. For systems with multiple relays, a relay selection approach was commonly used such that only one relay was active for each round of transmission [8-10]. In this work, we consider a two-user TWRN with two relay nodes (RNs), as shown in Fig. 1. Unlike the existing works that the users in a NOMA pair are connected to the same relay, the two terminals associated with the two different relays form a NOMA pair. Compared with one-way relaying with one active relay node, the terminals, relays and the BS will suffer from serve inter-stream interference in the considered TWRN, due to the increased number of data streams. 
To this end, we design an efficient NOMA-based relaying scheme that only three time slots are required for each round of transmission, as shown in Fig. 1. The proposed scheme can simultaneously provide wireless connectivity to isolated users with no connections to the BS, thereby improving spectral utilization. The achievable sum-rates for the proposed scheme with both AF and DF relaying are analyzed. For AF-based NOMA TWR scheme, referred to as NOMA-AF, a closed-form expression for the ergodic sum-rate upper bound is derived. For DF-based NOMA TWR scheme, referred to as NOMA-DF, we derived the exact ergodic sumrate. We further study the performance of the proposed NOMA-AF and NOMA-DF schemes in the high signal-to-noise ratio (SNR) region. Numerical results demonstrated the accuracy of the analytical results. It is shown that the sum-rate of the schemes is independent of the transmit power of the relays in the high SNR regions, given the transmit powers of the BS and users.

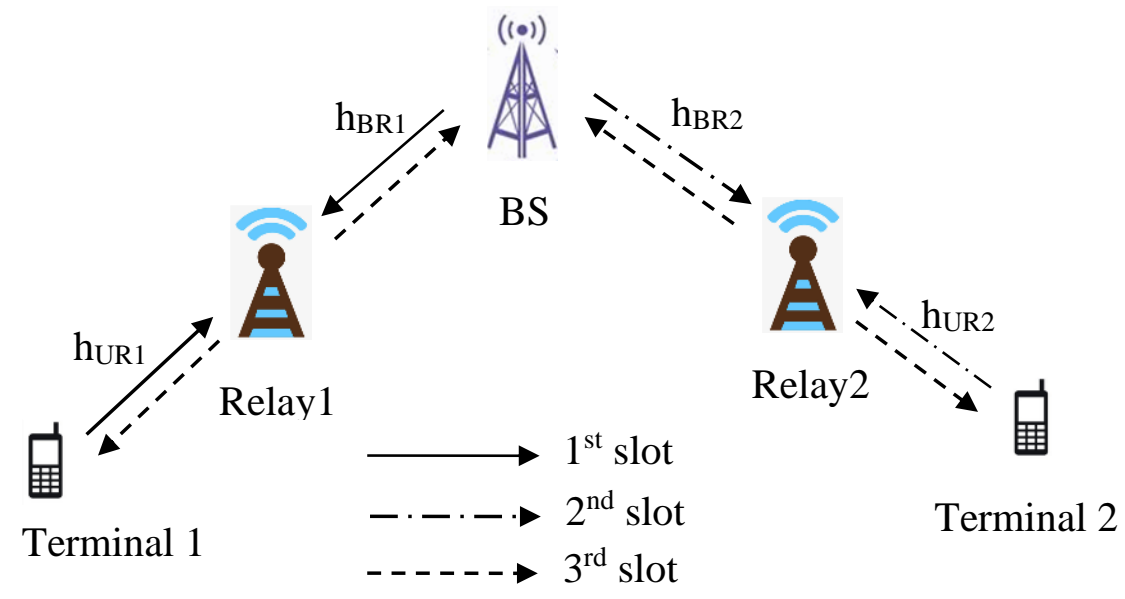

Fig. 1. Two-way relaying based on NOMA

\section{System Model}

Fig. 1 shows a TWRN consist of one BS, two terminals and two relays. It is assumed that both two terminals are far from the BS and the direct links are unavailable. Let $h_{B R, k}$ be the channel between the BS and relay $R_{k}, k=1,2$, and $h_{U R, k}$ be the channel between terminal $U_{k}$ and relay $R_{k}$. In this work, we assume time division duplex (TDD) that the channels between any two nodes are reciprocal. The channels remain the same during a transmission block, but may vary from block to block independently [4-7]. We also assume that terminal $U_{1}\left(\right.$ or $U_{2}$ ) is far away from the relay $R_{2}$ (or $R_{1}$ ), and no direct link exists between them. This is often the case, as the two relays are usually deployed with a large distance apart and buildings between terminal $U_{1}$ (or $U_{2}$ ) and the relay $R_{2}$ (or $R_{1}$ ) can block their radio propagation to each other.

For conventional TDMA-based transmissions, four time slots are usually needed for the considered TWRN, as two slots are used for each terminal to exchange messages with the BS. In this work, we design a NOMA-based TWR scheme that can reduce the number of required time slots into three. Specifically, in the first time slot, the BS and $U_{1}$ send signals to relay $R_{1}$. In the second time slot, the $\mathrm{BS}$ and $U_{2}$ send signals to relay $R_{2}$ simultaneously, while the two relays broadcast the signals to the BS and the terminalsat the third slot. 


\subsection{The First Time Slot}

The transmit signal of the BS is given by $x_{B, 1}=\sqrt{P_{B}} S_{B, 1}$, where $P_{B}$ is the power budget of the BS, and $s_{B, 1}$ is the symbol to be send to terminal $U_{1}$. The transmit signal of $U_{1}$ is given by $x_{U, 1}=\sqrt{P_{1}} s_{U, 1}$, where $P_{1}$ is the transmit power. As mentioned earlier, the BS and terminal $U_{1}$ send signals to $R_{1}$ in the first slot. The received signals at $R_{1}$ is given by

$$
y_{R, 1}=h_{B R, 1} x_{B, 1}+h_{U R, 1} x_{U, 1}+n_{R, 1}
$$

where $n_{R, 1} \sim C N\left(0, \sigma^{2}\right)$ denotes the additive white Gaussian noise (AWGN) at $R_{1}$.

\subsection{The Second Time Slot}

Both the BS and terminal $U_{2}$ send signals to $R_{2}$ in the second slot, while terminal $U_{1}$ and relay $R_{2}$ keep idle. The received signals at relay $R_{2}$ is given by

$$
y_{R, 2}=h_{B R, 2} x_{B, 2}+h_{U R, 2} x_{U, 2}+n_{R, 2}
$$

where $\mathrm{x}_{\mathrm{B}, 2}=\sqrt{\mathrm{P}_{\mathrm{B}}} \mathrm{s}_{\mathrm{B}, 2}$ is transmit signal of the $\mathrm{BS}, \mathrm{x}_{\mathrm{U}, 2}=\sqrt{\mathrm{P}_{2}} \mathrm{~s}_{\mathrm{U}, 2}$ is the transmit signal of terminal $\mathrm{U}_{2}$, and $\mathrm{n}_{\mathrm{R}, 2} \sim C N\left(0, \sigma^{2}\right)$ is the AWGN at relay $\mathrm{R}_{2}$.

\subsection{The Third Time Slot}

In the third time slot, let $x_{R, k}$ be the transmit signal of relay $R_{k}$ with a power of $\alpha_{k} P_{R}, k=$ 1,2 , where $\alpha_{1}$ and $\alpha_{2}$ are power allocation factors with $\alpha_{1}+\alpha_{2}=1$ and $P_{R}$ denotes the total power budget of the two relays. As mentioned before, the two relays broadcast the signals to the BS and the two terminals. The received signals at the BS and the two terminals are respectively given by

and

$$
y_{B}=\sum_{k=1}^{2} h_{B R, k} x_{R, k}+n_{B}
$$

$$
y_{U, k}=h_{U R, k} x_{R, k}+n_{U, k}, k=1,2,
$$

where $n_{B} \sim C N\left(0, \sigma^{2}\right)$ and $n_{U, k} \sim C N\left(0, \sigma^{2}\right)$ are AWGNs.

\section{AF-based NOMA Two-Way Relaying}

\subsection{The NOMA-AF Scheme}

For NOMA-AF, the transmit signal at $R_{k}$ in the third slot is generated by

$$
x_{R, k}=\theta_{k} y_{R, k}, k=1,2 \text {, }
$$

where $\theta_{k}$ is the amplification coefficient. $\theta_{k}$ can be calculated as 


$$
\theta_{k}=\sqrt{\frac{\alpha_{k} P_{R}}{\left|h_{B R, k}\right|^{2} P_{B}+\left|h_{U R, k}\right|^{2} P_{k}+\sigma^{2}}} .
$$

\subsubsection{Uplink of NOMA-AF}

Substituting (1), (2), (5) and (6) into (3), $y_{B}$ at the BS is given by

$$
y_{B}=\sum_{k=1}^{2} \theta_{k} h_{B R, k}\left(h_{B R, k} x_{B, k}+h_{U R, k} x_{U, k}+n_{R, k}\right)+n_{B} .
$$

Note that the signal related to $x_{B, k}$ in the right hand side (RHS) of (7) is the self-interference known by the BS, and can be removed completely. After removing the self-interference, the signals at the BS can be expressed by

$$
\begin{aligned}
\tilde{y}_{B} & =y_{B}-\sum_{k=1}^{2} \theta_{k} h_{B R, k}^{2} x_{B, k} \\
& =\theta_{1} h_{B R, 1} h_{U R, 1} x_{U, 1}+\theta_{2} h_{B R, 2} h_{U R, 2} x_{U, 2}+\tilde{n}_{B}
\end{aligned}
$$

where $\tilde{n}_{B}=\sum_{k=1}^{2} \theta_{k} h_{B R, k} n_{R, k}+n_{B}$ is the total noise with a variance of $\tilde{\sigma}_{B}^{2}=$ $\sum_{k=1}^{2}\left|\theta_{k} h_{B R, k}\right|^{2} \sigma^{2}+\sigma^{2}$. Assuming that $\alpha_{1}>\alpha_{2}$, i.e., the transmit power of $R_{1}$ is higher than $R_{2}$, the BS detects the signal $s_{U, 1}$ first by treating $s_{U, 2}$ as noise. Then, $s_{U, 1}$ will be subtracted from $\tilde{y}_{B}$ to detect $s_{U, 2}$. The achievable uplink rates of the two terminals are respectively given by

$$
R_{U L, 1}^{A F}=\frac{1}{3} \log _{2}\left(1+\frac{\left|\theta_{1} h_{U R, 1} h_{B R, 1}\right|^{2} P_{1}}{\left|\theta_{2} h_{U R, 2} h_{B R, 2}\right|^{2} P_{2}+\widetilde{\sigma}_{B}^{2}}\right)
$$

and

$$
R_{U L, 2}^{A F}=\frac{1}{3} \log _{2}\left(1+\frac{\left|\theta_{2} h_{U R, 2} h_{B R, 2}\right|^{2} P_{2}}{\widetilde{\sigma}_{B}^{2}}\right)
$$

\subsubsection{Downlink of NOMA-AF}

From (1), (2), (5) and (6), the received signal at terminal $U_{k}$ is

$$
y_{U, k}=\theta_{k} h_{U R, k}\left(h_{B R, k} x_{B, k}+h_{U R, k} x_{U, k}+n_{R, k}\right)+n_{U, k},
$$

Note that the signal $x_{U, k}$ in the RHS of (11) is the self-interference, which can be removed completely. After removing the self-interference, $U_{k}$ is able to detect the desired signal directly. The achievable downlink rate of $U_{k}$ is

$$
R_{D L, k}^{A F}=\frac{1}{3} \log _{2}\left(1+\frac{\left|\theta_{k} h_{B R, k} h_{U R, k}\right|^{2} P_{B}}{\left|\theta_{k} h_{U R, k}\right|^{2} \sigma^{2}+\sigma^{2}}\right) .
$$

Remark 1: In the NOMA-AF scheme, SIC is employed at the BS only. The signal detection complexity at the terminals and relays are the same as in conventional TDMA-based transmission scheme.

\subsection{Sum-Rate of NOMA-AF}




\subsubsection{Sum-Rate Upperbound of NOMA-AF}

Define $\rho_{k}=P_{k} / \sigma^{2}, k=1,2, \rho_{R}=P_{R} / \sigma^{2}$, and $\rho_{B}=P_{B} / \sigma^{2}$. Let $G_{B R, k}=E\left(\left|h_{B R, k}\right|^{2}\right)$ denote the average gain of the channel between the BS and $R_{k}, k=1,2$, and $G_{U R, k}=$ $E\left(\left|h_{U R, k}\right|^{2}\right)$ be the average channel gain between relay $R_{k}$ and terminal $U_{k}, k=1,2$. By averaging over all possible channel realizations, we have the following result on the ergodic sum-rate of the proposed NOMA-AF scheme.

Proposition 1: The ergodic sum-rate of NOMA-AF is upper bounded by:

$$
\begin{aligned}
& \tilde{R}_{S u m}^{A F, U B}=\sum_{k=1}^{2} \frac{\log _{2}(e)}{3} \Phi\left(\frac{1}{\alpha_{k} \rho_{R} G_{U R, k}}+\frac{1}{\mu_{k} \rho_{B} G_{B R, k}}\right) \\
& +\frac{\log _{2}(e)}{3\left(1-c_{1}\right)}\left(\Phi\left(\frac{1}{\alpha_{1} \mu_{3} \rho_{1} G_{U R, 1}}\right)-\Phi\left(\frac{1}{\alpha_{1} \mu_{3} \rho_{1} G_{U R, 1} c_{1}}\right)\right) \\
& +\frac{\log _{2}(e)}{3} \Phi\left(\frac{1}{\mu_{4} \rho_{2} G_{U R, 2}}+\frac{1}{\mu_{4} \rho_{B} G_{B R, 2}}\right),
\end{aligned}
$$

where $\Phi(x) \triangleq e^{x} E_{1}(x), E_{1}(x)$ is the exponential integral function, $\mu_{k}=\alpha_{k} \rho_{R} /\left(\alpha_{k} \rho_{R}+\right.$ $\left.\rho_{k}\right), k=1,2, \mu_{3}=\rho_{R} /\left(\rho_{B}+\rho_{R}\right), \mu_{4}=\alpha_{2} \rho_{R} /\left(\rho_{B}+\rho_{R}\right)$, and $c_{1}=\alpha_{2} \rho_{2} G_{U R, 2} / \alpha_{1} \rho_{1} G_{U R, 1}$.

Proof: See Appendix A.

\subsubsection{Asymptotic Sum-Rate Upper bound of NOMA-AF}

As will be shown later, the derived upper bound in (13) is tight. In the following, we further investigate the asymptotic sum-rate performance of NOMA-AF in the high power (SNR) range. Note that $E_{1}(x) \simeq-C_{0}-\ln (x)$ and $e^{x} \simeq 1$ as $x \rightarrow 0$, where $C_{0}=0.5772 \ldots$ is the Euler constant $[26]$ and $(1,5,11)$. We have

$$
\Phi(x)=e^{x} E_{1}(x) \simeq-C_{0}-\ln (x), x \rightarrow 0 .
$$

As a result, the asymptotic sum-rate upper bound (13) for NOMA-AF can be expressed as

$$
\begin{aligned}
& \tilde{R}_{\text {sum }}^{A F, U B} \simeq \frac{1}{3\left(1-c_{1}\right)} \log _{2}\left(\frac{1}{c_{1}}\right)-\log _{2}(e) C_{0} \\
& -\sum_{k=1}^{2} \frac{1}{3} \log _{2}\left(\frac{1}{\alpha_{k} \rho_{R} G_{U R, k}}+\frac{1}{\mu_{k} \rho_{B} G_{B R, k}}\right) \\
& -\frac{1}{3} \log _{2}\left(\frac{1}{\mu_{4} \rho_{2} G_{U R, 2}}+\frac{1}{\mu_{4} \rho_{B} G_{B R, 2}}\right) .
\end{aligned}
$$

Remark 2: From (15), the sum-rate upper bound of NOMA-AF depends on the power allocation factors $\alpha_{1}$ and $\alpha_{2}$.

Remark 3: When the powers of the terminals and the BS are fixed, i.e., $P_{B}, P_{1}$, and $P_{2}$ are fixed, as $P_{R} \rightarrow+\infty$, we have $\mu_{k}=\alpha_{k} \rho_{R} /\left(\alpha_{k} \rho_{R}+\rho_{k}\right) \simeq 1, k=1,2, \mu_{3}=\rho_{R} /\left(\rho_{B}+\right.$ $\left.\rho_{R}\right) \simeq 1$, and $\mu_{4} \simeq \alpha_{2}$. Then, the asymptotic sum-rate upper bound in (15) can be written as 


$$
\begin{aligned}
& \tilde{R}_{\text {Sum }}^{A F, U B} \simeq \frac{1}{3\left(1-c_{1}\right)} \log _{2}\left(\frac{1}{c_{1}}\right)-\log _{2}(e) C_{0} \\
& -\sum_{k=1}^{2} \frac{1}{3} \log _{2}\left(\frac{1}{\rho_{B} G_{B R, k}}\right) \\
& -\frac{1}{3} \log _{2}\left(\frac{1}{\alpha_{2} \rho_{2} G_{U R, 2}}+\frac{1}{\alpha_{2} \rho_{B} G_{B R, 2}}\right),
\end{aligned}
$$

which is a constant independent of $P_{R}$. This suggests that increasing the power budget of the relays only can not always improve the sum-rate of NOMA-AF.

\section{DF-based NOMA Two-Way Relaying}

\subsection{The NOMA-DF Scheme}

In NOMA-DF, the two relays first decode the received signal with SIC. Take the decoding operation at relay $R_{1}$ as an example. Since the BS's transmit power is usually higher than the terminal $U_{1}, R_{1}$ detects $s_{B, 1}$ first by treating $s_{U, 1}$ as noise. Then, $s_{B, 1}$ is removed from $y_{R, 1}$ to detect $s_{U, 1}$. The detection process of relay $R_{2}$ is the same as that of $R_{1}$.

From (1) and (2), the achievable uplink rate from terminal $U_{k}$ to the relay $R_{k}$ is

$$
R_{U L, k}^{U_{k}, R_{k}}=\frac{1}{3} \log _{2}\left(1+\frac{\left|h_{U R, k}\right|^{2} P_{k}}{\sigma^{2}}\right),
$$

provided that $s_{B, k}$ was successfully decoded, i.e.,

$$
R_{D L, k}^{B, R_{k}} \leq \frac{1}{3} \log _{2}\left(1+\frac{\left|h_{B R, k}\right|^{2} P_{B}}{\left|h_{U R, k}\right|^{2} P_{k}+\sigma^{2}}\right)
$$

After decoding, the relay $R_{k}$ broadcasts signal $x_{R, k}=\sqrt{\alpha_{k} P_{R}} S_{R, k}$ to the BS and $U_{k}$ in the third slot, where $s_{R, k}=s_{B, k} \oplus s_{U, k}$ is the XOR encoded signal.

\subsubsection{Uplink of NOMA-DF}

In the third slot, the signal $y_{B}$ at the BS for NOMA-DF is

$$
y_{B}=\sum_{k=1}^{2} h_{B R, k} \sqrt{\alpha_{k} P_{R}} s_{R, k}+n_{B} .
$$

As in the NOMA-AF scheme, the BS detects $s_{R, 1}$ first, then $s_{R, 1}$ is removed to detect $s_{R, 2}$. The data rate for the link from $R_{2}$ to the $\mathrm{BS}$ given by

$$
R_{U L, 2}^{R_{2}, B}=\frac{1}{3} \log _{2}\left(1+\frac{\alpha_{2} P_{R}\left|h_{B R, 2}\right|^{2}}{\sigma^{2}}\right)
$$

under the constraint that the rate from $R_{1}$ to the BS satisfies

$$
R_{U L, 1}^{R_{1}, B} \leq \frac{1}{3} \log _{2}\left(1+\frac{\alpha_{1} P_{R}\left|h_{B R, 1}\right|^{2}}{\alpha_{2} P_{R}\left|h_{B R, 2}\right|^{2}+\sigma^{2}}\right)
$$




\subsubsection{Downlink of NOMA-DF}

For the down link transmission from $R_{k}$ to terminal $U_{k}$, from (4), we have

$$
R_{D L, k}^{R_{k}, U_{k}}=\frac{1}{3} \log _{2}\left(1+\alpha_{k} P_{R}\left|h_{U R, k}\right|^{2} / \sigma^{2}\right), \quad k=1,2 .
$$

Now consider the achievable rates for the proposed NOMA-DF scheme. From (17) and (21), the uplink rate of terminal $U_{1}$ is given by

where

$$
R_{U L, 1}^{D F}=\frac{1}{3} \log _{2}\left(1+\gamma_{U L, 1}^{D F}\right)
$$

$$
\gamma_{U L, 1}^{D F}=\min \left(P_{1}\left|h_{U R, 1}\right|^{2} / \sigma^{2}, \frac{\alpha_{1} P_{R}\left|h_{B R, 1}\right|^{2}}{\alpha_{2} P_{R}\left|h_{B R, 2}\right|^{2}+\sigma^{2}}\right)
$$

Similarly, from (17) and (20), the uplink rate of $U_{2}$ is given by

where

$$
R_{U L, 2}^{D F}=\frac{1}{3} \log _{2}\left(1+\gamma_{U L, 2}^{D F}\right),
$$

$$
\gamma_{U L, 2}^{D F}=\min \left(P_{2}\left|h_{U R, 2}\right|^{2} / \sigma^{2}, \alpha_{2} P_{R}\left|h_{B R, 2}\right|^{2} / \sigma^{2}\right)
$$

For downlink transmission, from (18) and (22), we have

where

$$
R_{D L, k}^{D F}=\frac{1}{3} \log _{2}\left(1+\gamma_{D L, k}^{D F}\right),
$$

$$
\gamma_{D L, k}^{D F}=\min \left(\frac{\left|h_{B R, k}\right|^{2} P_{B}}{\left|h_{U R, k}\right|^{2} P_{k}+\sigma^{2}}, \frac{\alpha_{k} P_{R}\left|h_{U R, k}\right|^{2}}{\sigma^{2}}\right) .
$$

Remark 4: In NOMA-DF, SIC is employed at the BS and the relays. The signal detection at the two terminals is as low as in TDMA-based schemes.

\subsection{Sum-Rate Performance of NOMA-DF}

\subsubsection{The Exact Sum-Rate of NOMA-DF}

Proposition 2: By averaging over all possible channel realizations, the ergodic sum-rate of the proposed NOMA-DF scheme for the TWRN is given by:

$$
\begin{aligned}
& \tilde{R}_{\text {sum }}^{D F}=\frac{\log _{2}(e)}{3\left(1-c_{2}\right)}\left(\Phi\left(\psi_{1}\right)-\Phi\left(\frac{\psi_{1}}{c_{2}}\right)\right)+\frac{\log _{2}(e)}{3} \Phi\left(\psi_{2}\right) \\
& +\sum_{k=1}^{2} \frac{\log _{2}(e)}{3\left(1-d_{k}\right)}\left(\Phi\left(\eta_{k}\right)-\Phi\left(\frac{\eta_{k}}{d_{k}}\right)\right),
\end{aligned}
$$


where $c_{2}=\alpha_{2} G_{B R, 2} / \alpha_{1} G_{B R, 1}, \psi_{k}=\frac{1}{\alpha_{k} \rho_{R} G_{B R, k}}+\frac{1}{\rho_{k} G_{U R, k}}, \eta_{k}=\frac{1}{\rho_{B} G_{B R, k}}+\frac{1}{\alpha_{k} \rho_{R} G_{U R, k}}$, and $d_{k}=$ $\rho_{k} G_{U R, k} / \rho_{B} G_{B R, k}, k=1,2$.

Proof: See Appendix B.

\subsubsection{The Asymptotic Sum-Rate of NOMA-DF}

Consider the asymptotic performance in the high SNR region. Using the result in (14), the asymptotic sum-rate of NOMA-DF is

$$
\begin{aligned}
\tilde{R}_{\text {sum }, \text { asym }}^{\text {DF }} & =\frac{\log _{2}(e)}{3\left(1-c_{2}\right)} \ln \left(\frac{1}{c_{2}}\right)-\frac{\log _{2}(e)}{3}\left(C_{0}+\ln \left(\psi_{2}\right)\right) \\
& -\sum_{k=1}^{2} \frac{\log _{2}(e)}{3\left(1-d_{k}\right)}\left(C_{0}+\ln \left(\eta_{k}\right)+\Phi\left(\frac{\eta_{k}}{d_{k}}\right)\right) .
\end{aligned}
$$

Remark 5: From (30), the sum-rate of NOMA-DF also depends on the power allocation factors $\alpha_{1}$ and $\alpha_{2}$, as in NOMA-AF.

Remark 6: When the powers of the terminals and the $\mathrm{BS}$ are fixed, i.e., $P_{B}, P_{1}$, and $P_{2}$ are fixed, as $P_{R} \rightarrow+\infty$, we have $\psi_{k} \simeq \frac{1}{\rho_{k} G_{U R, k}}$, and $\eta_{k} \simeq \frac{1}{\rho_{B} G_{B R, k}}, k=1,2$. Then, the asymptotic sum-rate of NOMA-DF is given by

$$
\begin{aligned}
& \tilde{R}_{\text {Sum,asym }}^{\text {DF }}=\frac{\log _{2}(e)}{3\left(1-c_{2}\right)} \ln \left(\frac{1}{c_{2}}\right)-\frac{\log _{2}(e)}{3} C_{0} \\
& -\frac{\log _{2}(e)}{3} \ln \left(\frac{1}{\rho_{2} G_{U R, 2}}\right)-\sum_{k=1}^{2} \frac{\log _{2}(e)}{3\left(1-d_{k}\right)} C_{0} \\
& -\sum_{k=1}^{2} \frac{\log _{2}(e)}{3\left(1-d_{k}\right)}\left(\ln \left(\frac{1}{\rho_{B} G_{B R, k}}\right)+\Phi\left(\frac{1}{d_{k} \rho_{B} G_{B R, k}}\right)\right) .
\end{aligned}
$$

From (31), we conclude that the asymptotic sum-rate of NOMA-DF approaches to a constant as in NOMA-AF scheme in this case.

\section{Simulation Results}

During the simulations, the channels are independent Rayleigh distributed, and the average channel gains are $G_{B R, 1}=G_{B R, 2}=0 \mathrm{~dB}, G_{U R, 1}=-5 \mathrm{~dB}$, and $G_{U R, 2}=-10 \mathrm{~dB}$. Unless otherwise specified, the power allocation factors are chosen as $\alpha_{1}=0.9$, and $\alpha_{2}=0.1$. The powers of the two terminals are the same, i.e., $P_{1}=P_{2}=P$. The noise variance is $\sigma^{2}=0$ $\mathrm{dBm}$. The transmit powers for the BS and the two relays are given in the following.

In Fig. 2, we plot the sum-rates of the proposed NOMA-AF and NOMA-DF schemes. The $\mathrm{SNR}$ in the figure is defined as $S N R=P / \sigma^{2}$. The powerof the BS is $30 \mathrm{~dB}$ higher than that of the terminals, while the total power of the relays is $20 \mathrm{~dB}$ higher. For NOMA-AF, the sumrate upper bound (13) and the asymptotic upper bound (15) are also shown in the figure. While for NOMA-DF, we plot the analytical ergodic sum-rate (29) and the asymptotic sum-rate (30). From the figure, we see that the derived upper bound is tight across the whole SNR region for NOMA-AF, and the asymptotic upper bound for NOMA-AF is tight. For NOMA-DF, the analyzed exact and asymptotic ergodic sum-rates are also accurate. It can also be seen that a 
much higher sum-rate can be achieved for NOMA-DF as compared with NOMA-AF in the whole SNR range.

Fig. 3 compares the performance of the proposed schemes with conventional TDMA-based TWR transmissions. While Fig. 4 shows the individual terminal rates of these schemes. Note that in TDMA-based AF and DF relaying schemes (labeled as "TDMA-AF" and "TDMA-DF" in the figure), four time slots are required for information exchange between the two terminals and the BS. From Fig. 3, we see that much higher sum-rates can be achieved by the NOMAAF and NOMA-DF schemes as compared with TDMA-AF and TDMA-DF. For instance, a sum-rate gain of $30 \%$ is observed for NOMA-AF as compared with TDMA-AF scheme when the SNR is above $10 \mathrm{~dB}$. For the individual rates shown in Fig. 4, we see that terminal $U_{1}$ achieves a higher data rate for the proposed schemes in the whole SNR region. While for terminal $U_{2}$, the proposed schemes outperform TDMA-based schemes when the SNR is above $15 \mathrm{~dB}$.

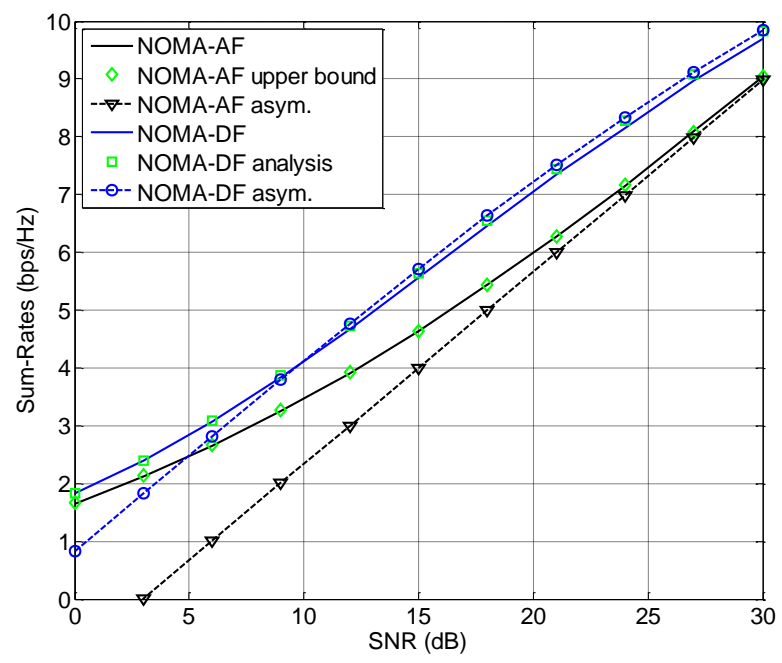

Fig. 2. Sum-rate performance of the NOMA-AF and NOMA-DF schemes

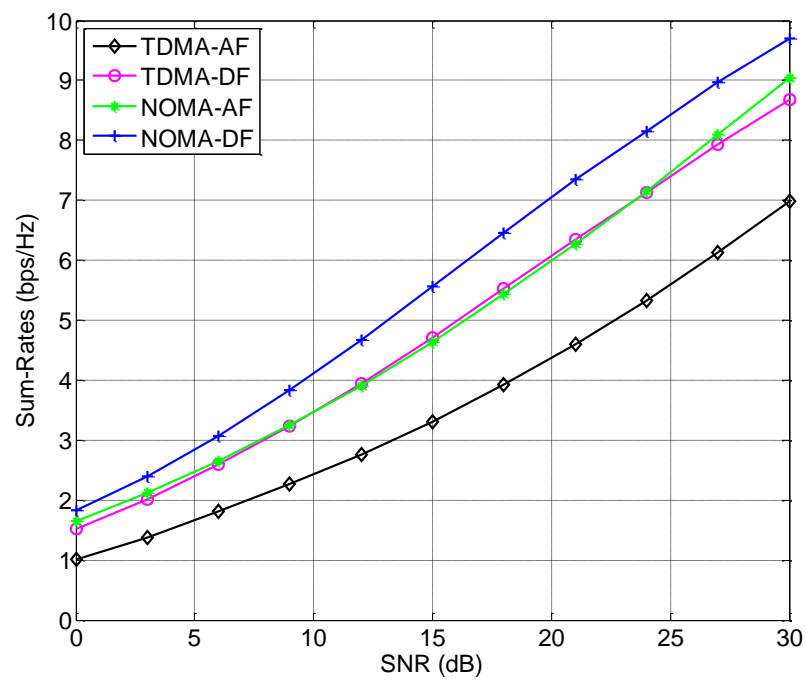

Fig. 3. Sum-rates performance comparison between NOMA and TDMA-based schemes 


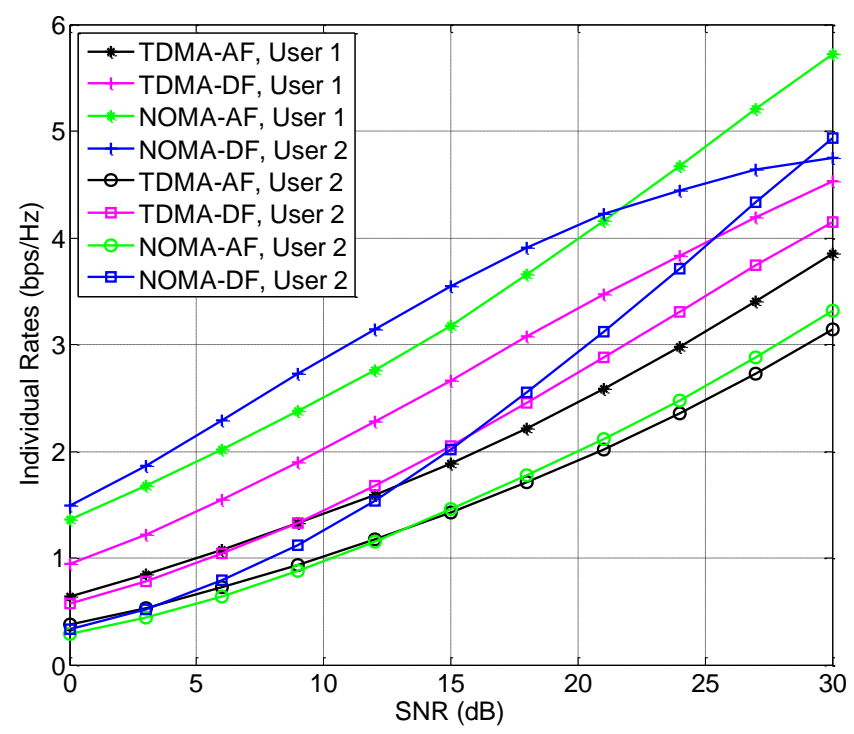

Fig. 4. Individual rates comparison between NOMA and TDMA-based schemes

Next, Fig. 5 investigates the impact of the relay's power budget on NOMA-AF and NOMADF. Here, the transmit power of the BS is fixed to be $P_{B}=40 \mathrm{dBm}$, and the powers of the two terminals are $P_{1}=P_{2}=10 \mathrm{dBm}$, respectively. From the figure, we see that the derived upper bound for NOMA-AF is tight, and the analyzed sum-rate for NOMA-DF is accurate. When the power budget of the relays is smaller than $50 \mathrm{dBm}$, the achievable sum-rates of both schemes increase with the relay's transmit power. However, the sum-rates approach to certain constants in the high power region for both NOMA-AF and NOMA-DF, which agrees with the analytical results in Remarks 3 and 6.

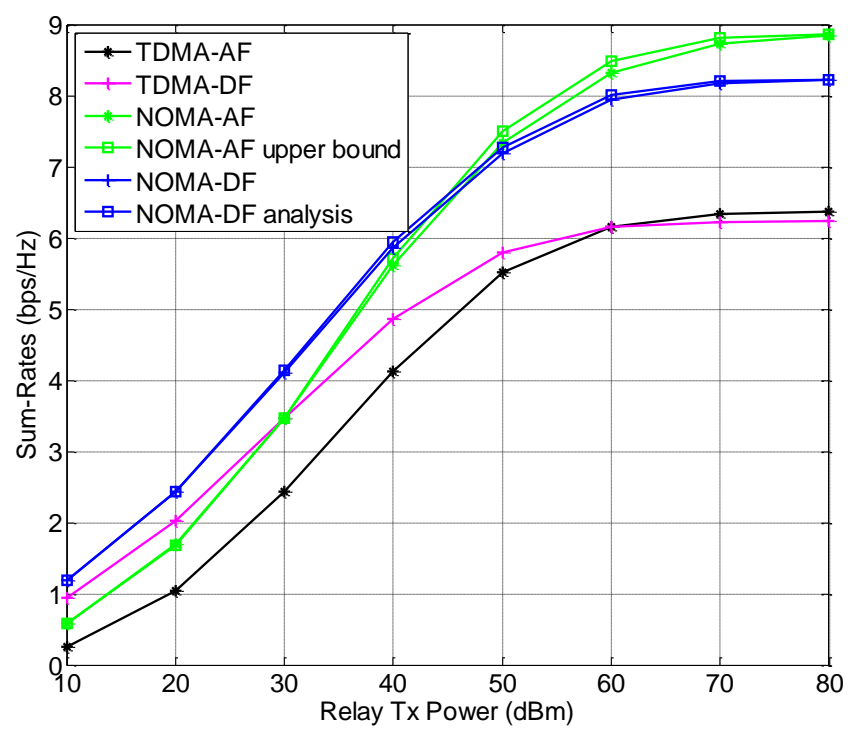

Fig. 5. Sum-rates under different relay power budgets 
Similar results can be observed in Fig. 6, which shows the performance of NOMA-AF and NOMA-DF with different BS power budgets. The powers of the relay stations and the two terminals are fixed to be $P_{R}=30 \mathrm{dBm}$ and $P_{1}=P_{2}=10 \mathrm{dBm}$, respectively. The NOMA-DF scheme outperforms NOMA-AF when $P_{B} \geq 25 \mathrm{dBm}$. It can be seen that there is an optimal BS power value to maximize the sum-rates of NOMA-AF. This is due to the fact that the downlink rate of NOMA-AF increases with the transmit power of the BS, while the uplink rate decreases with $P_{B}$ for lowtomoderate BS power budgets, see $(9,10,12)$. While for high BS power budgets, both the uplink and downlink rates approach to certain constants. For NOMA-DF, the achievable downlink rate increases while the uplink data rates remain constant for low to moderate BS power budgets. Hence, the sum-rate of NOMA-DF increases with the transmit power of the BS when $P_{B}<40 \mathrm{dBm}$. The sum-rates of both schemes approach to certain constants in the high BS power region.

Finally, Fig. 7 investigates the sum-rates under different power allocation factors $\alpha_{1}$. In the simulations, we $\operatorname{set} P_{B}=40 \mathrm{dBmand} P_{1}=P_{2}=10 \mathrm{dBm}$. While the total power of the two relays is set to be $P_{R}=15$ or $30 \mathrm{dBm}$. From the figure, we see that when $P_{R}=15 \mathrm{dBm}$, the achievable sum-rates of both NOMA-AF and NOMA-DF remain almost unchanged. However, when $P_{R}=30 \mathrm{dBm}$, we see that the sum-rates of both schemes depend on $\alpha_{1}$. The individual rates of the proposed schemes with different $\alpha_{1}$ are shown in Fig. 8, where $P_{B}=40 \mathrm{dBm}$, $P_{R}=30 \mathrm{dBm}$, and $P_{1}=P_{2}=10 \mathrm{dBm}$. From the figure, it is clear that as $\alpha_{1}$ becomes large, more power is allocated to relay $R_{1}$, which results in a higher rate for terminal $U_{1}$, while the data rate of terminal $U_{2}$ decreases.

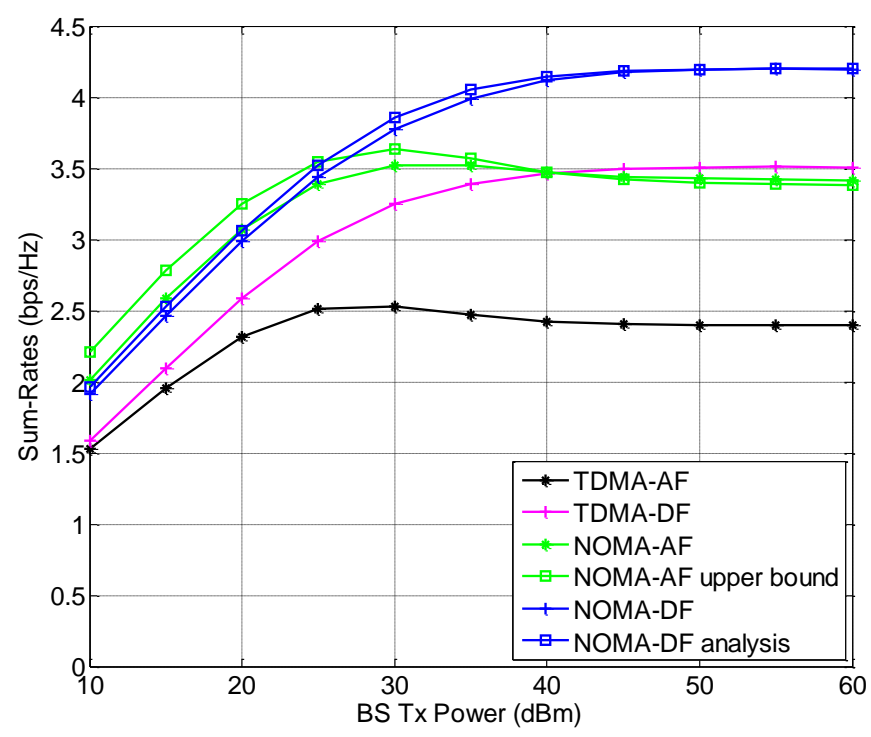

Fig. 6. Sum-rates under different BS power budgets 


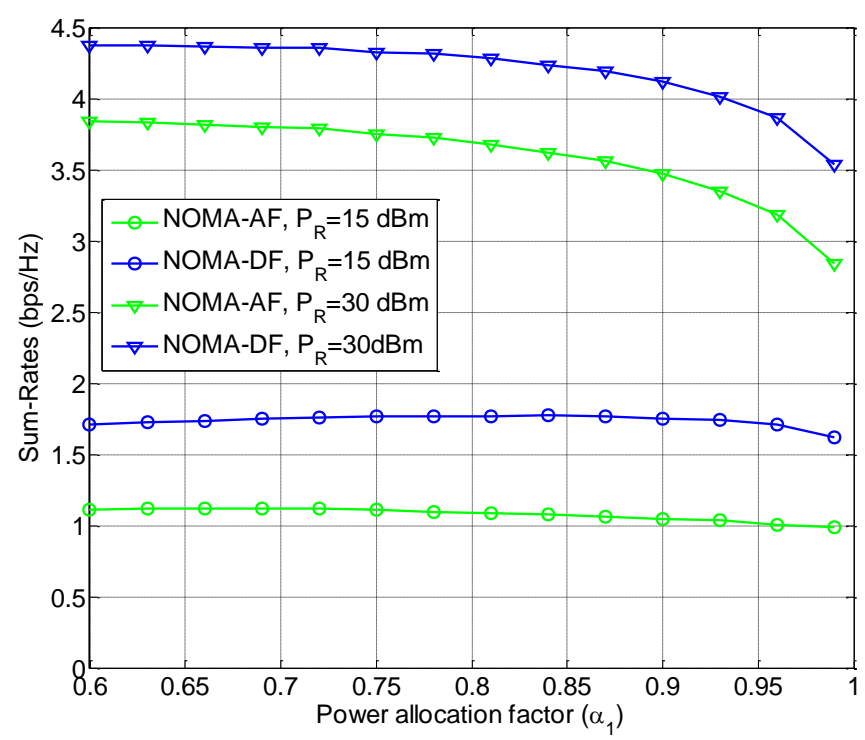

Fig. 7. Sum-rates under different power allocation factors $\left(\alpha_{1}\right), P_{B}=40 \mathrm{dBm}$ and $P_{1}=P_{2}=10$ $\mathrm{dBm}$

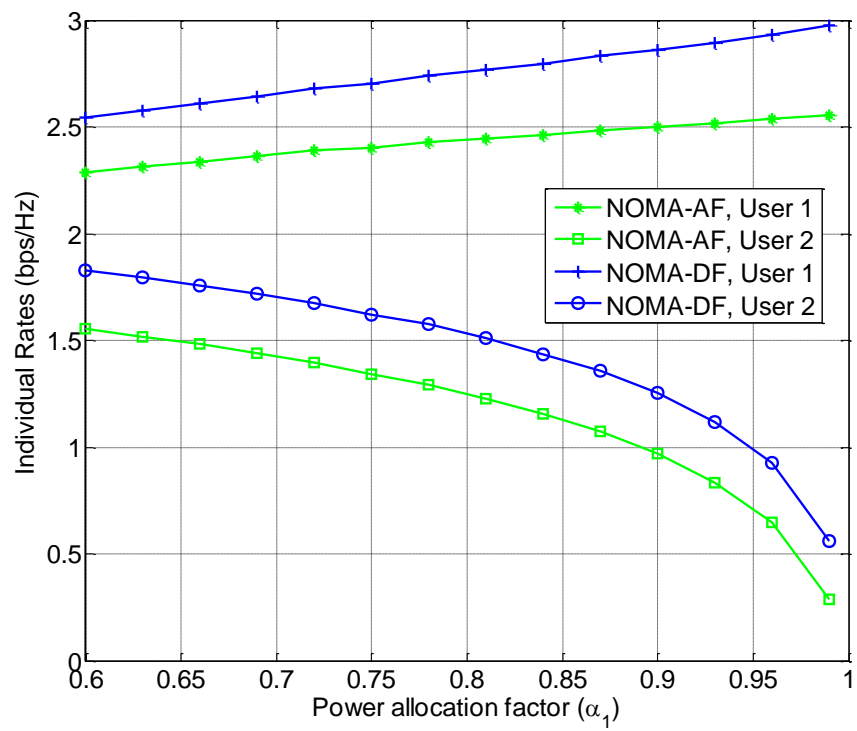

Fig. 8. Individual rates under different power allocation factors $\left(\alpha_{1}\right), P_{B}=40 \mathrm{dBm}, P_{1}=P_{2}=10$ $\mathrm{dBm}$, and $P_{R}=30 \mathrm{dBm}$

\section{Conclusion}

This paper designed efficient NOMA-based TWR schemes for a two-terminal two-relay system. With the proposed NOMA-AF and NOMA-DF schemes, the BS and the terminals are able to exchange messages within three time slots. We analyzed the sum-rates of NOMA-AF and NOMA-DF, and derived a closed-form upper bound for NOMA-AF, and the exact ergodic 
sum-rate of NOMA-DF. We further investigated the asymptotic sum-rates of NOMA-AF and NOMA-DF in the high SNR region. We reveal that the sum-rates of the proposed NOMAbased TWR schemes are independent of the transmit power of the relays in the high SNR region. Simulation results showed that NOMA-AF and NOMA-DF outperform the existing TDMA-based schemes significantly.

\section{Appendix A: Proof of Proposition 1}

For the downlink transmission of $U_{1}$, the received SINR at terminal $U_{k}, k=1,2$, can be expressed as

$$
\begin{aligned}
\gamma_{D L, k}^{A F} & =\frac{\alpha_{k}\left|\theta_{k} h_{B R, k} h_{U R, k}\right|^{2} \rho_{B}}{\left|\theta_{k} h_{U R, k}\right|^{2+1}} \\
& =\frac{\alpha_{k} \rho_{R} \rho_{B}\left|h_{B R, k}\right|^{2}\left|h_{U R, k}\right|^{2}}{\left(\rho_{k}+\alpha_{k} \rho_{R}\right)\left|h_{U R, k}\right|^{2}+\rho_{B}\left|h_{B R, k}\right|^{2}+1},
\end{aligned}
$$

which can be upper bounded by

$$
\gamma_{D L, k}^{A F} \leq \gamma_{D L, k}^{A F, U B}=\mu_{k} \min \left(\left(\alpha_{k} \rho_{R}+\rho_{k}\right)\left|h_{U R, k}\right|^{2}, \rho_{B}\left|h_{B R, k}\right|^{2}\right),
$$

where $\mu_{k}=\alpha_{k} \rho_{R} /\left(\alpha_{k} \rho_{R}+\rho_{k}\right), k=1,2$.

The CDF of the SINR upper bound $\gamma_{D L, k}^{A F, U B}$ is

$$
F_{\gamma_{D L, k}^{A F, U B}}(x)=1-e^{-\frac{x}{\alpha_{k} \rho_{R} G_{U R, k}}} e^{-\frac{x}{\mu_{k} \rho_{B} G_{B R, k}}}
$$

As a result, the downlink rate upper bound of terminal $U_{k}$ can be calculated as

$$
\begin{aligned}
\tilde{R}_{D L, k}^{A F, U B} & =\int_{0}^{+\infty} \frac{1}{3} \log _{2}(1+x) f_{\gamma_{D L, k}^{A F, U B}}(x) d x \\
& =\frac{\log _{2}(e)}{3} \int_{0}^{+\infty} \frac{1-F}{\gamma_{D L, k}^{A F, U B}(x)} d x \\
& =\frac{\log _{2}(e)}{3} \Phi\left(\frac{1+x}{\alpha_{k} \rho_{R} G_{U R, k}}+\frac{1}{\mu_{k} \rho_{B} G_{B R, k}}\right)
\end{aligned}
$$

where $\Phi(x)=e^{x} E_{1}(x), E_{1}(x)$ is the exponential integral function, and the last step is due to $\int_{0}^{+\infty} e^{-a x} /(b+x) d x=e^{a b} E_{1}(a b)$ as in [26] and $(1,5,28)$.

Now consider the uplink transmission rate of terminal $U_{1}$. Note that in the second step we have used the approximation that $\left|\theta_{k} h_{B R, k}\right|^{2} \simeq \frac{\alpha_{k} P_{R}}{P_{B}}$ as $P_{B} \gg P_{k}, k=1,2$ holds for practical systems. Then, the received SINR for $U_{1}$ at the BS can be expressed as

$$
\begin{aligned}
\gamma_{U L, 1}^{A F} & =\frac{\left|\theta_{1} h_{U R, 1} h_{B R, 1}\right|^{2} P_{1}}{\left|\theta_{2} h_{U R, 2} h_{B R, 2}\right|^{2} P_{2}+\widetilde{\sigma}_{B}^{2}} \\
& =\frac{\alpha_{1} \mu_{3} \rho_{1}\left|h_{U R, 1}\right|^{2}}{\alpha_{2} \mu_{3} \rho_{2}\left|h_{U R, 2}\right|^{2}+1}
\end{aligned}
$$


where $\mu_{3}=\rho_{R} /\left(\rho_{B}+\rho_{R}\right)$. The $\mathrm{CDF}$ of $\gamma_{U L, 1}^{A F}$ is

$$
F_{\gamma_{U L, 1}^{A F}}(x)=1-\frac{1}{1+c_{1} x} e^{-\frac{x}{\alpha_{1} \mu_{3} \rho_{1} G_{U R, 1}}}
$$

where $c_{1}=\alpha_{2} \rho_{2} G_{U R, 2} / \alpha_{1} \rho_{1} G_{U R, 1}$. Hence, the uplink rate of terminal $U_{1}$ is given by

$$
\begin{aligned}
& \tilde{R}_{U L, 1}^{A F}=\int_{0}^{+\infty} \frac{1}{3} \log _{2}(1+x) f_{\gamma_{U L, 1}^{A F}}(x) d x \\
& =\frac{\log _{2}(e)}{3} \int_{0}^{+\infty} \frac{1}{(1+x)\left(1+c_{1} x\right)} e^{-\frac{x}{\alpha_{1} \mu_{3} \rho_{1} G_{U R, 1}}} d x \\
& =\frac{\log _{2}(e)}{3\left(1-c_{1}\right)}\left(\Phi\left(\frac{1}{\alpha_{1} \mu_{3} \rho_{1} G_{U R, 1}}\right)-\Phi\left(\frac{1}{\alpha_{1} \mu_{3} \rho_{1} G_{U R, 1} c_{1}}\right)\right) .
\end{aligned}
$$

For the uplink transmission of $U_{2}$, the received SINR can be expressed as

$$
\begin{aligned}
\gamma_{U L, 2}^{A F} & \simeq \frac{\left|\theta_{2} h_{U R, 2} h_{B R, 2}\right|^{2} \rho_{2}}{1+\frac{P_{R}}{P_{B}}} \\
& =\mu_{4} \frac{\rho_{2} \rho_{B}\left|h_{U R, 2}\right|^{2}\left|h_{B R, 2}\right|^{2}}{\rho_{2}\left|h_{U R, 2}\right|^{2}+\rho_{B}\left|h_{B R, 2}\right|^{2}+1},
\end{aligned}
$$

where $\mu_{4}=\alpha_{2} \rho_{R} /\left(\rho_{B}+\rho_{R}\right)$.

Using the inequality that $\frac{X Y}{X+Y+1} \leq \min (X, Y)$ for any non-negative variables $X$ and $Y$, the uplink received SINR of terminal $U_{2}$ is upper bound by

$$
\gamma_{U L, 2}^{A F} \leq \gamma_{U L, 2}^{A F, U B}=\mu_{4} \min \left(\rho_{2}\left|h_{U R, 2}\right|^{2}, \rho_{B}\left|h_{B R, 2}\right|^{2}\right),
$$

whose CDF is given by

$$
F_{\gamma_{U L, 2}^{A F, U B}}(x)=1-e^{-\frac{x}{\mu_{4} \rho_{2} G} U R, 2} e^{-\frac{x}{\mu_{4} \rho_{B} G_{B R, 2}}}
$$

Then, the upper bound for the data rate of $U_{2}$ in the uplink is

$$
\begin{aligned}
& \tilde{R}_{U L, 2}^{A F, U B}=\int_{0}^{+\infty} \frac{1}{3} \log _{2}(1+x) f_{\gamma_{U L, 2}^{A F, U B}}(x) d x \\
& =\frac{\log _{2}(e)}{3} \int_{0}^{+\infty} \frac{1}{1+x} e^{-\frac{x}{\mu_{4} \rho_{2} G_{U R, 2}} e^{-\frac{x}{\mu_{4} \rho_{B} G_{B R, 2}}}} d x \\
& =\frac{\log _{2}(e)}{3} \Phi\left(\frac{1}{\mu_{4} \rho_{2} G_{U R, 2}}+\frac{1}{\mu_{4} \rho_{B} G_{B R, 2}}\right) .
\end{aligned}
$$

From (35), (38), and (42), we obtain the sum-rate upper bound in Proposition 1. 


\section{Appendix B: Proof of Proposition 2}

For terminal $U_{1}$, the CDF of $\gamma_{1}=\frac{\left|h_{B R, 1}\right|^{2} \alpha_{1} \rho_{R}}{\left|h_{B R, 2}\right|^{2} \alpha_{2} \rho_{R}+1}$ can be calculated as

$$
F_{\gamma_{1}}(x)=1-\frac{1}{1+c_{2} x} e^{-\frac{x}{\alpha_{1} \rho_{R} G B R, 1}}
$$

where $c_{2}=\alpha_{2} G_{B R, 2} / \alpha_{1} G_{B R, 1}$. Then, the CDF of $\gamma_{U L, 1}^{D F}=\min \left(\gamma_{2}, \gamma_{1}\right)$ is

$$
F_{\gamma_{U L, 1}^{D F}}(x)=1-\frac{1}{1+c_{2} x} e^{-\frac{x}{\alpha_{1} \rho_{R} G_{B R, 1}}} e^{-\frac{x}{\rho_{1} G_{U R, 1}}}
$$

Based on (44), the uplink data rate of $U_{1}$ is given by

$$
\begin{aligned}
& \tilde{R}_{U L, 1}^{D F}=\int_{0}^{+\infty} \frac{1}{3} \log _{2}(1+x) f_{\gamma_{U L, 1}^{D F}}(x) d x \\
& =\frac{\log _{2}(e)}{3} \int_{0}^{+\infty} \frac{1}{(1+x)\left(1+c_{2} x\right)} e^{-\psi_{1} x} d x \\
& =\frac{\log _{2}(e)}{3\left(1-c_{2}\right)}\left(\Phi\left(\psi_{1}\right)-\Phi\left(\frac{\psi_{1}}{c_{2}}\right)\right),
\end{aligned}
$$

where $\psi_{1}=\frac{1}{\alpha_{1} \rho_{R} G_{B R, 1}}+\frac{1}{\rho_{1} G_{U R, 1}}$.

For terminal $U_{2}$ in the uplink, the CDF of $\gamma_{U L, 2}^{D F}=\min \left(\rho_{2}\left|h_{U R, 2}\right|^{2}, \alpha_{2} \rho_{R}\left|h_{B R, 2}\right|^{2}\right)$ is given by

$$
F_{\gamma_{U L, 2}^{D F}}(x)=1-e^{-\frac{x}{\rho_{2} G_{U R, 2}}} e^{-\frac{x}{\alpha_{2} \rho_{R} G_{B R, 2}}}
$$

The uplink data rate of $U_{2}$ is given by

where $\psi_{2}=\frac{1}{\rho_{2} G_{U R, 2}}+\frac{1}{\alpha_{2} \rho_{R} G_{B R, 2}}$.

$$
\begin{aligned}
\tilde{R}_{U L, 2}^{D F} & =\int_{0}^{+\infty} \frac{1}{3} \log _{2}(1+x) f_{\gamma_{U L, 2}^{D F}}(x) d x \\
& =\frac{\log _{2}(e)}{3} \Phi\left(\psi_{2}\right),
\end{aligned}
$$

For $U_{k}, k=1,2$ in the downlink, note that the $\mathrm{CDF}$ of $\frac{\left|h_{B R, k}\right|^{2} \rho_{B}}{\left|h_{U R, k}\right|^{2} \rho_{k}+1}$ is given by

$$
F_{k}(x)=1-\frac{1}{1+d_{k} x} e^{-\frac{x}{\rho_{B} G_{B R, k}}},
$$

where $=\rho_{k} G_{U R, k} / \rho_{B} G_{B R, k}, k=1,2$. Hence, the $\operatorname{CDF}$ of $\gamma_{D L, k}^{D F}$ is then given by

where $\eta_{k}=\frac{1}{\rho_{B} G_{B R, k}}+\frac{1}{\alpha_{k} \rho_{R} G_{U R, k}}$.

$$
F_{\gamma_{D L, k}^{D F}}(x)=1-\frac{1}{1+d_{k} x} e^{-\eta_{k} x},
$$


The achievable downlink rate of $U_{k}$ is now upper bounded by

$$
\begin{aligned}
& \tilde{R}_{D L, k}^{D F}=\int_{0}^{+\infty} \frac{1}{3} \log _{2}(1+x) f_{\gamma_{D L, K}^{D F}}(x) d x \\
& =\frac{\log _{2}(e)}{3} \int_{0}^{+\infty} \frac{1}{(1+x)\left(1+d_{k} x\right)} e^{-\eta_{k} x} d x \\
& =\frac{\log _{2}(e)}{3\left(1-d_{k}\right)}\left(\Phi\left(\eta_{k}\right)-\Phi\left(\frac{\eta_{k}}{d_{k}}\right)\right) .
\end{aligned}
$$

Based on (45), (47) and (50), we can obtain the result in Proposition 2.

\section{References}

[1] S. Li and Y. Sun, "Power allocation for full-duplex NOMA relaying based underlay D2D communications," KSII Transactions on Internet and Information Systems, vol. 13, no. 1, pp. 1633, 2019. Article (CrossRef Link)

[2] T. Huynh, P. N. Son, and M. Voznak, "Exact Outage Probability of Two-Way Decode-andForward NOMA Scheme with Opportunistic Relay Selection," KSII Transactions on Internet and Information Systems, vol. 13, no. 12, pp. 5862-5887, 2019. Article (CrossRef Link)

[3] Y. Saito, Y. Kishiyama, A. Benjebbour, T. Nakamura, A. Li, and K. Higuchi, "Non-orthogonal multiple access (NOMA) for cellular future radio access," in Proc. of IEEE $77^{\text {th }}$ Vehicular Technology Conference, pp. 1-5, June 2013. Article (CrossRef Link)

[4] Z. Ding, M. Peng, and H. V. Poor, "Cooperative non-orthogonal multiple access in 5G systems," IEEE Communications Letters, vol. 19, no. 8, pp. 1462-1465, Aug. 2015. Article (CrossRef Link)

[5] X. Yue, Y. Liu, S. Kang, A. Nallanathan, and Z. Ding, "Exploiting Full/Half-duplex user relaying in NOMA systems," IEEE Transactions Communications, vol. 66, no. 2, pp. 560-575, Feb. 2018. Article (CrossRef Link)

[6] J. B. Kim and I. H. Lee, "Non-orthogonal multiple access in coordinated direct and relay transmission," IEEE Communications Letters, vol. 19, no. 11, pp. 2037-2040, Nov. 2015. Article (CrossRef Link)

[7] X. Liang, Y. Wu, D. W. K. Ng, Y. Zuo, S. Jin, and H. Zhu, "Outage performance for cooperative NOMA transmission with an AF relay," IEEE Communications Letters, vol. 21, no. 11, pp. 24282431, 2017. Article (CrossRef Link)

[8] P. Xu, Z. Yang, Z. Ding, and Z. Zhang, "Optimal relay selection schemes for cooperative NOMA," IEEE Transactions on Vehicular Technology, vol. 67, no. 8, pp. 7851-7855, Aug. 2018. Article (CrossRef Link)

[9] Z. Yang, Z. Ding, Y. Wu, and P. Fan, "Novel relay selection strategies for cooperative NOMA," IEEE Transactions on Vehicular Technology, vol. 6, no. 11, pp. 10114-10123, Nov. 2017. Article (CrossRef Link)

[10] Y. Li, Y. Li, X. Chu, Y. Ye, and H. Zhang, "Performance analysis of relay selection in cooperative NOMA networks," IEEE Communications Letters, vol. 23, no. 4, pp. 760-763, Apr. 2019. Article (CrossRef Link)

[11] W. Xiaoxiang, Z. Jia, and W. DongYu, "Joint Relay-and-Antenna Selection and Power Allocation for AF MIMO Two-way Relay Networks," KSII Transactions on Internet and Information Systems, vol. 10, no. 3, pp. 1016-1033, 2016. Article (CrossRef Link)

[12] S. Lou and L. Yang, "Opportunistic Multiple Relay Selection for Two-Way Relay Networks with Outdated Channel State Information," KSII Transactions on Internet and Information Systems, vol. 8, no. 2, pp. 389-405, 2014. Article (CrossRef Link)

[13] Z. Fang, F. Liang, S. Zhang, and J. Shi, "Protocol design and data detection for two-way relay networks with fraction asynchronous delays," International Journal of Communication Systems, vol. 27, no. 10, pp. 1984-1993, Oct. 2014. Article (CrossRef Link) 
[14] M. K. Shukla, H. H. Nguyen, and O. J. Pandey, "Secrecy performance analysis of two-way relay non-orthogonal multiple access systems," IEEE Access, vol. 8, pp. 39502-39512, 2020. Article (CrossRef Link)

[15] W. Zhao, R. She, and H. Bao, "Security energy efficiency maximization for two-way relay assisted cognitive radio NOMA network with self-interference harvesting," IEEE Access, vol. 7, pp. 7440174411, 2019. Article (CrossRef Link)

[16] X. Yue, Y. Liu, S. Kang, A. Nallanathan, and Y. Chen, "Modeling and analysis of two-way relay non-orthogonal multiple access systems," IEEE Transactions Communications, vol. 66, no. 9, pp. 3784-3796, Sep. 2018. Article (CrossRef Link)

[17] F. Wei, T. Zhou, T. Xu, and H. Hu, "Modeling and analysis of two-way relay networks: A joint mechanism using NOMA and network coding," IEEE Access, vol. 7, pp. 152679-152689, 2019. Article (CrossRef Link)

[18] X. Tang, K. An, K. Guo, S. Wang, X. Wang, J. Li, and F. Zhou, "On the performance of two-way multiple relay non-orthogonal multiple access-based networks with hardware impairments," IEEE Access, vol. 7, pp. 128896-128909, 2019. Article (CrossRef Link)

[19] X. Wang, M. Jia, I. W. H. Ho, Q. Guo, and F. C. Lau, "Exploiting full-duplex two-way relay cooperative non-orthogonal multiple access," IEEE Transactions Communications, vol. 67, no. 4, pp. 2716-2729, 2019. Article (CrossRef Link)

[20] B. Zheng, X. Wang, M. Wen, and F. Chen, "NOMA-Based multi-pair two-way relay networks with rate splitting and group decoding," IEEE Journal on Selected Areas in Communications, vol. 35, no. 10, pp. 2328-2341, Oct. 2017. Article (CrossRef Link)

[21] C. Y. Ho and C. Y. Leow, "Cooperative non-orthogonal multiple access with physical layer network coding," IEEE Access, vol. 7, pp. 44894-44902, 2019. Article (CrossRef Link)

[22] C. Zhang and X. Jia, "Joint beamforming optimisation for NOMA-based wireless powered multipair two-way AF and DF relaying networks," IET Communications, vol. 13, no. 4, pp. 387-395, Mar. 2019. Article (CrossRef Link)

[23] S. Silva, G. A. A. Baduge, M. Ardakani, and C. Tellambura, "NOMA-Aided multi-way massive MIMO relay," IEEE Transactions on Communications, vol. 68, no. 7, pp. 4050-4062, 2020. Article (CrossRef Link)

[24] X. Li, Q. Wang, Y. Liu, T. A. Tsiftsis, Z. Ding, and A. Nallanathan, "UAV-Aided multi-way NOMA networks with residual hardware impairments," IEEE Wireless Communications Letters, vol. 9, no. 9, pp. 1538-1542, 2020. Article (CrossRef Link)

[25] Z. Fang, J. Li, and Y. Lu, "Cooperative non-orthogonal multiple access for two-way relay networks," AEU International Journal of Electronics and Communications, vol. 115, 2020. Article (CrossRef Link)

[26] M. Abramowitz, and I. A. Stegun, Handbook of Mathematical Functions with Formulas, Graphs, and Mathematical Tables, $9^{\text {th }}$ ed., New York, NY, USA, 1970. Article (CrossRef Link)

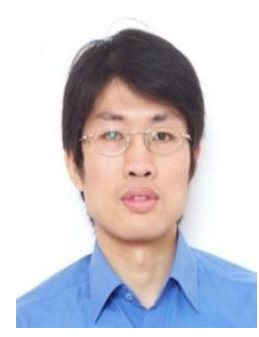

Guosheng Li received the B. E. degree in computer science from Hubei University of Technology in 2000, and the M. S. degree in software engineering from Hangzhou Dianzi University in 2004. He is a Lecture in the School of Intelligent Information Engineering, Zhejiang Wanli University, Ningbo, China. His research interests include mobile communications, embedded systems and mobile applications developing. 